\title{
Evaluation of Variable Parameter MCV Control Chart in Downward Process Shifts
}

\author{
XINYING CHEW \\ School of Computer Sciences \\ Universiti Sains Malaysia \\ 11800 Penang \\ MALAYSIA
}

\begin{abstract}
Previous studies revealed that the coefficient of variation (CV) is important in ensuring process quality, especially for monitoring a process where its process mean and variance are highly correlated. The fact that almost all industrial process monitoring involves a minimum of two or more related quality characteristics being monitored simultaneously. The existing adaptive charts for monitoring the multivariate $\mathrm{CV}$ are focused on detecting upward process shifts. The downward process monitoring is crucial since it shows process improvement. Very little research works are available on the downward adaptive multivariate CV chart. This makes it difficult for the quality engineer who wishes to implement the adaptive multivariate $\mathrm{CV}$ chart on the downward process monitoring. Therefore, this paper filled the research gap by proposing a downward variable parameter chart for the multivariate coefficient of variation. The performance measures of the proposed charts are derived based on the Markov-chain approach. Numerical comparisons between the proposed and existing charts have been made, in terms of the average time to signal criterion. The findings reveal that the proposed charts outperform the existing charts for detecting small and moderate downward process shifts.
\end{abstract}

Key-words: Statistics, downward shifts, Markov-chain, variable parameter, control charting technique Received: January 16, 2021. Revised: July 23, 2021. Accepted: August 18, 2021. Published: September 5, 2021.

\section{Introduction}

Control charting techniques are commonly used and received great attention to their application in various industries, such as manufacturing healthcare, chemical, biological, agriculture and service industries. The traditional $\bar{X}$ and $R$ charts could not be used for some processes especially when the mean and standard deviation of the processes are highly correlated. In this case, the traditional control charts may provide erroneous conclusions. To deal with this kind of process, the implementation of a coefficient of variation $(\mathrm{CV})$ control chart is the best solution. In the past few decades, the application of $\mathrm{CV}$ is very common to be applied in various disciplines. This can be shown in some recent research works. Whelan and Siqueira [1] applied the CV in crop area classification across multiple climates. Weber [2] investigated the $\mathrm{CV}$ as the predictor of risk sensitivity. Chanda et al. [3] presented the use of $\mathrm{CV}$ on the sugarcane population and stalk production. Additionally, the CV can be implemented to evaluate the global solar radiation in terms of time scale separation [4] and chemical reactor process [5].

Kang et al [6] were the pioneer to introduce a standard CV chart. Subsequently, research works on $\mathrm{CV}$ charts have been extensively conducted to improve the sensitivity of the standard CV chart, in terms of detecting small and moderate $\mathrm{CV}$ shifts. such as those by $[7-10]$, to name a few. Besides, Yeong et al. [11] introduced a multivariate CV (MCV) chart for multivariate process monitoring. Khaw et al. $[12,13]$ suggested the adaptive MCV and synthetic MCV charts enhance the statistical performance of the standard MCV chart of Yeong et al. [11]. Nguyen et al. [14] and Chew and Khaw [15] proposed the onesided MCV charts by incorporating the variable sampling interval (VSI) and variable sample size and sampling interval (VSSI), respectively. Chew et al. [16] discussed the upward MCV chart with variable parameters. In addition, a fixed and variable exponentially weighted moving average (EWMA) chart for monitoring the MCV was presented by GinerBosch et al. [17] and Ayyoub et al. [18], respectively.

The adaptive scheme is a well-known scheme in the control charting technique, which consists of VSI, variable sample size (VSS), VSSI and variable parameter (VP) strategies. Among those strategies, VP is known as the most effective adaptive scheme. The VP strategy is continuously being investigated by many researchers. Yeong et al. [19] recommended a VP chart for monitoring the CV. Lee [20] and Lin and Chou [21] used a VP chart to monitor the autocorrelated and non-normality processes, 
respectively. Moreover, the VP charts with correlated A\&L switching rule [22], VP $\bar{X}$ chart [23] and VP $S$ chart [24] were investigated in terms of economic criterion. More recently, Sabahno et al. [25] considered the VP chart for simultaneously monitoring the process mean and variability with measurement error while the VP auxiliary information based chart was developed using a Markov-chain approach [26].

The VP MCV chart of Chew et al. [16] showed an outstanding performance in the detection of $\mathrm{MCV}$ shifts when compared to other existing MCV charts. However, a setback of this study is the VP MCV chart was only considered for the detection of upward MCV shifts. In most of the scenarios, the detection of downward MCV shifts is important as it shows process improvement. With the intention to fill the research gap in downward process monitoring and the excellent feature of the VP scheme, this paper extends the VP MCV chart of Chew et al. [16] and proposes a onesided downward VP (DVP MCV) chart for monitoring the downward MCV shifts. Note that the one-sided DVP MCV chart can avoid the biased average time to signal (ATS) performance. In the existing literature, only the Shewhart (SH) and VSSI MCV charts are available for monitoring the downward process shifts. The SH MCV chart can detect large process shifts quickly but it is rather slow in the detection of small and moderate process shifts. The statistical sensitivity of the SH MCV chart can be enhanced by using a variable parameter strategy. This paper proposes a one-sided downward VP MCV (called DVP MCV) chart. The performance measures of the proposed chart are derived using the Markov-chain approach. As it will be shown in Section 3, the DVP MCV chart generally outperforms the existing DSH MCV and DVSSI MCV charts, in the detection of small and moderate shifts, in terms of the ATS criterion.

The remainder sections are organized as follows: Section 2 discusses the design of the one-sided DVP MCV charts. The derivations of the formulae and algorithms to compute the ATS values, by adopting the Markov-chain method are also provided. Numerical comparisons among the DVP MCV, DVSSI MCV and DSH MCV charts are enumerated in Section 3. Lastly, the research findings and recommendations for future research are given in the last section.

\section{One-sided Downward Variable Parameter MCV Chart}

Let $\boldsymbol{X}_{1}, \boldsymbol{X}_{2}, \ldots, \boldsymbol{X}_{n}$ refers to a multivariate $n_{0}$ from the $p$-variate normal distribution with $\mu$ and $\Sigma$. Voinov and Nikulin [27] derived the population MCV statistic as $\gamma=\left(\boldsymbol{\mu}^{T} \boldsymbol{\Sigma}^{-1} \boldsymbol{\mu}\right)^{-\frac{1}{2}}$, where $\boldsymbol{\mu}$ and $\boldsymbol{\Sigma}$ refer to the mean vector and covariance matrix, respectively. Note that $\gamma$ can be estimated by the sample MCV, $\hat{\gamma}$ when $\mu$ and $\Sigma$ are unspecified. Thus, $\hat{\gamma}=\left(\overline{\boldsymbol{X}}^{T} \boldsymbol{S}^{-1} \overline{\boldsymbol{X}}\right)^{-\frac{1}{2}}$ by replacing $\mu$ and $\boldsymbol{\Sigma}$ with $\overline{\boldsymbol{X}}$ and $\boldsymbol{S}$, respectively. Here, $\overline{\boldsymbol{X}}$ is the sample mean vector whereas $\boldsymbol{S}$ is the sample covariance matrix. The computations of $\bar{X}$ and $\boldsymbol{S}$ are $\overline{\boldsymbol{X}}=1 / n \sum \boldsymbol{X}$ and $\boldsymbol{S}=1 /(n-1) \sum(\boldsymbol{X}-\overline{\boldsymbol{X}})(\boldsymbol{X}-\overline{\boldsymbol{X}})^{T}$, respectively, where $\bar{X}$ and $S$ are independent of one another.

The DSH MCV chart of Yeong et al. [11] uses a fixed sampling interval, $h_{0}$, fixed sample size, $n_{0}$ and fixed-width constant. It consists of two regions, i.e. the safe region and the action region. Contrary to the DSH MCV chart, the DVP MCV chart contains three regions, which represent the safe, warning and action regions. The VP scheme varies the sampling interval, sample size and width constant to enhance the flaw of the DSH MCV chart in the detection of small and moderate downward MCV shifts. The sampling interval adopted for the proposed chart is varied between the short sampling interval, $h_{S}$ and the long sampling interval, $h_{L}$, where $h_{S}<\mathrm{ASI}_{0}<h_{L}$ while the sample size is varied between the small sample size, $n_{S}$ and the large sample size, $n_{L}$, where $n_{S}<\mathrm{ASS}_{0}<n_{L}$. Note that $\mathrm{ASI}_{0}$ and $\mathrm{ASS}_{0}$ refer to the in-control average sampling interval (ASI) and incontrol average sample size, respectively, where $\mathrm{ASI}_{0}$ and $\mathrm{ASS}_{0}$ of the DVP MCV chart is equal to the fixed sampling interval and fixed sample size of the DSH MCV for ensuring a fair comparison can be made. The DVP MCV chart works as follows:

i) When the $t$-th sample MCV, $\hat{\gamma}_{t}$ falls above the warning limit, the process shows no indication of trouble. Hence, $h_{L}$ ,$n_{S}$ and loosened control and warning limits are taken to compute the $(t+1)$-th sample MCV, $\hat{\gamma}_{t+1}$;

ii) When $\hat{\gamma}_{t}$ falls below the warning limit and above the control limit, the process still shows no indication of trouble but there is a higher tendency for it to become out-of-control. Hence, $h_{S}, n_{L}$ and tightened control and warning limits are taken to compute $\hat{\gamma}_{t+1}$; and

iii) When $\hat{\gamma}_{t}$ falls below the control limit, the process shows an indication of trouble at the $t$-th sample due to the presence of 
assignable causes. In this case, an immediate investigation should be taken.

Subsequently, the lower warning and control limits of the DVP MCV chart are computed as

$\mathrm{LWL}_{k}=F_{\hat{\gamma}}^{-1}\left(\alpha_{k}^{\prime} \mid n_{r}, p, \delta_{0}\right)$,

and

$\mathrm{LCL}_{k}=F_{\hat{\gamma}}^{-1}\left(\alpha_{k} \mid n_{r}, p, \delta_{0}\right)$.

Note that in Eqns (1) and (2), when $k=S$, then $n_{r}=n_{L}$. Conversely, when $k=L$, then $n_{r}=n_{S}$. The Type-I error probability, $\alpha_{0}$ is set based on the desired in-control ATS $\left(\operatorname{ATS}_{0}\right)$ and $\alpha_{k}^{\prime}>\alpha_{0}$. The Type-I error probability is used in the computation of lower control limit, i.e. LCL. The LCL is computed according to the specified $\mathrm{ATS}_{0}$ value, where the Type-I error probability is set to fulfil this constraint. $\mathrm{LWL}_{S}$, $\mathrm{LCL}_{S}, \mathrm{LWL}_{L}$ and $\mathrm{LCL}_{L}$ denote the tightened warning limit, tightened control limit, loosened warning limit and loosened control limit, respectively, where $\mathrm{LCL}_{S}<\mathrm{LCL}_{0}<\mathrm{LCL}_{L}$. Here, $\mathrm{LCL}_{0}$ is the lower control limit of the non-adaptive MCV chart and can be obtained using Eqn (2) by substituting $\alpha_{k}$ as $\alpha_{0}$. Here, $\delta_{0}=n / \gamma_{0}^{2}$ and $p$ denotes the number of quality characteristics.

Equations (1) and (2) follow an inverse cumulative distribution function (cdf) of $\hat{\gamma}$, i.e. $F_{\hat{\gamma}}^{-1}(\alpha \mid n, p, \delta)=$ $\sqrt{[(n(n-p)) /((n-1) p)] \times\left(1 / F_{F}^{-1}(1-\alpha \mid p, n-p, \delta)\right)}$. Note that $F_{F}^{-1}(\cdot)$ refers to an inverse cdf of a noncentral $F$ distribution, where this distribution is only valid when $p<n$ due to the degree of freedom and $\delta$ is defined as $n /\left(\tau \gamma_{0}\right)^{2}$, where the shift size $\tau=1$ when the process is in-control. Note that $\gamma_{1}=\tau \gamma_{0}$ is the out-of-control $\mathrm{MCV}$, where $\tau \neq 1$, with the values of $0<\tau<1$ refer to downward MCV shifts.

The Markov chain model of the DVP MCV chart has three states, which correspond to the safe, warning and action regions. States 1 and 2 are the transient states (i.e. $A_{11}, A_{12}, A_{21}$ and $A_{22}$ ) while state 3 is the absorbing state, where $\mathrm{A}_{\mathrm{ij}}$ is the transition probability from the previous state $i$ to the current state $j$. Then, the transition probabilities of the transient states in the matrix can be obtained as

$A_{11}=\operatorname{Pr}\left(\hat{\gamma} \geq \mathrm{LWL}_{L} \mid n_{S}, p, \delta_{11}\right)=1-F_{\hat{\gamma}}\left(\mathrm{LWL}_{L} \mid n_{S}, p, \delta_{11}\right)$

, where $\delta_{11}=\frac{n_{S}}{\gamma_{1}^{2}}$

$$
\begin{aligned}
& A_{12}=\operatorname{Pr}\left(\mathrm{LCL}_{L}<\hat{\gamma} \leq \mathrm{LWL}_{L} \mid n_{S}, p, \delta_{11}\right) \\
& =F_{\hat{\gamma}}\left(\mathrm{LWL}_{L} \mid n_{S}, p, \delta_{11}\right)-F_{\hat{\gamma}}\left(\mathrm{LCL}_{L} \mid n_{S}, p, \delta_{11}\right)
\end{aligned}
$$

$$
A_{21}=\operatorname{Pr}\left(\hat{\gamma} \geq \mathrm{LWL}_{S} \mid n_{L}, p, \delta_{12}\right)=1-F_{\hat{\gamma}}\left(\mathrm{LWL}_{S} \mid n_{L}, p, \delta_{12}\right)
$$

, where $\delta_{12}=\frac{n_{L}}{\gamma_{1}^{2}}$

$$
\begin{aligned}
& A_{22}=\operatorname{Pr}\left(\mathrm{LCL}_{S}<\hat{\gamma} \leq \mathrm{LWL}_{S} \mid n_{L}, p, \delta_{12}\right) \\
& =F_{\hat{\gamma}}\left(\mathrm{LWL}_{S} \mid n_{L}, p, \delta_{12}\right)-F_{\hat{\gamma}}\left(\mathrm{LCL}_{S} \mid n_{L}, p, \delta_{12}\right)
\end{aligned}
$$

The ATS of the DVP MCV chart can be computed as

$$
\operatorname{ATS}=\boldsymbol{b}^{T}(\boldsymbol{I}-\boldsymbol{Q})^{-1} \boldsymbol{t}
$$

$I$ and $Q$ are the identity and transient state transition probability matrices with $2 \times 2$ dimension, respectively, whereas $\boldsymbol{t}^{T}=\left(h_{2}, h_{1}\right)$ is a sampling intervals vector. Subsequently, $\boldsymbol{b}^{T}=\left(b_{1}, b_{2}\right)$ is the initial probability vector, subject to $b_{1}+b_{2}=1$. Here, $b_{1}$ and $b_{2}$ are the time spent proportions in the safe and warning regions, respectively. Both $b_{1}$ and $b_{2}$ are obtained based on $\tau=1$, where

$b_{1}=\frac{1-F_{\hat{\gamma}}\left(\mathrm{LWL}_{L} \mid n_{S}, p, \delta_{11}\right)}{1-F_{\hat{\gamma}}\left(\mathrm{LCL}_{L} \mid n_{S}, p, \delta_{11}\right)}$

And

$b_{2}=\frac{F_{\hat{\gamma}}\left(\mathrm{LWL}_{S} \mid n_{L}, p, \delta_{12}\right)-F_{\hat{\gamma}}\left(\mathrm{LCL}_{S} \mid n_{L}, p, \delta_{12}\right)}{1-F_{\hat{\gamma}}\left(\mathrm{LCL}_{S} \mid n_{L}, p, \delta_{12}\right)}$,

subject to the specified values of $\mathrm{ASS}_{0}, \mathrm{ASI}_{0}$ and $\mathrm{UCL}_{0}$, where $\mathrm{ASI}_{0}=h_{2} b_{1}+h_{1} b_{2}, \mathrm{ASS}_{0}=n_{1} b_{1}+n_{2} b_{2}$ and $\mathrm{UCL}_{0}=\mathrm{UCL}_{L} b_{1}+\mathrm{UCL}_{S} b_{2}$.

\section{Numerical Comparison}

The numerical performance of the proposed chart is evaluated in terms of the ATS criterion and the ATS is specified as 370. According to Muhammad et al. [28], an $\mathrm{ATS}_{0}$ of 370 ensures a low false alarm rate, Moreover, it also allows a fair comparison to be made with other MCV charts, i.e. DSH MCV, DVSI MCV, DVSS MCV and DVSSI MCV charts. The existing DSH MCV chart for monitoring the downward process has its fixed sampling interval set as, say $h_{0}=1$. As ATS $=h_{0} \times \mathrm{ATS}$, then ATS $=$ ARL for the 
proposed DVP MCV chart. For ensuring a fair comparison with the DSH MCV chart in terms of the ATS criterion, the $\operatorname{ASI}_{0}\left(=h_{0}\right)$ of the DVP MCV chart is set as unity. Then, the $h_{S}$ value is set to be at least 0.1 to minimize the $\mathrm{ATS}_{1}$ values, for detecting the decrease of MCV shifts. Tables $1-2$ present the comparison between the DVP MCV, DVSSI MCV [15], DVSS MCV [15], DVSI MCV [15] and DSH MCV [11] charts, in terms of the $\mathrm{ATS}_{1}$ criterion, for $p \in\{2,3\}, \gamma_{0} \in\{0.1,0.3,0.5\}, n \in\{5,10\}$ and $\tau \in\{0.50$, $0.60,0.70,0.80,0.90\}$. In the detection of downward MCV shifts, it is clear that the DVP MCV chart has the best performance, for small and moderate downward shift sizes. For example, from Tables 1 and 3 , when $p=2, n_{0}=5, \gamma_{0}=0.1$ and $\tau=0.50$, the DVP MCV, DVSSI MCV, DVSS MCV, DVSI MCV and DSH MCV charts provide $\mathrm{ATS}_{1}=2.09,2.56,4.69$, 6.18 and 33.43, respectively, where the DVP MCV chart has the smallest $\mathrm{ATS}_{1}$ value. Another example can be shown from Tables 2 and 4 , when $p=3, n_{0}=$ $10, \gamma_{0}=0.1$ and $\tau=0.70$, the DVP MCV, DVSSI $\mathrm{MCV}$ and DSH MCV charts give $\mathrm{ATS}_{1}=2.82,3.78$, 6.92, 7.05 and 44.02, respectively, where the DVP MCV chart has the smallest $\mathrm{ATS}_{1}$ value. In addition, from Tables 1 to 4, another notable trend observed is that the larger shift $\tau$ provides smaller TARL $\mathrm{T}_{1}$ values regardless of the sample size $n$ values. When $n$ and $\tau$ values are fixed, the TARL $L_{1}$ values increase consistently by increasing the $\gamma_{0}$ value. When $\gamma_{0}$ and $\tau$ values are fixed while the $n$ value increases, the TARL $_{1}$ value decreases. When the $p$ value increases, the TARL $L_{1}$ value increases as well, this shows that the $p$ value is proportional to the TARL $L_{1}$ value.

Table 1. ATS 1 values of the DVP MCV, DVSSI MCV and DSH MCV charts when $p=2, n_{0} \in\{5,10\}$, $\gamma_{0} \in\{0.1,0.3,0.5\}, h_{S}=0.1$ and $\mathrm{ATS}_{0}=370$.

\begin{tabular}{|c|c|c|c|}
\hline & DVP MCV & DVSSI MCV & DSH MCV \\
\hline & \multicolumn{3}{|c|}{$\gamma_{0}=0.1$} \\
\hline$\tau$ & \multicolumn{3}{|c|}{$n_{0}=5$} \\
\hline 0.5 & 2.09 & 2.56 & 33.43 \\
\hline 0.6 & 3.90 & 5.41 & 64.03 \\
\hline 0.7 & 9.41 & 15.52 & 103.67 \\
\hline 0.8 & 25.80 & 80.80 & 129.98 \\
\hline 0.9 & 102.31 & 203.13 & 237.24 \\
\hline \multicolumn{3}{|c|}{$n_{0}=10$} \\
\hline 0.5 & 1.13 & 1.18 & 5.28 \\
\hline 0.6 & 1.40 & 1.58 & 14.50 \\
\hline 0.7 & 2.40 & 3.12 & 36.56 \\
\hline 0.8 & 8.01 & 16.70 & 80.33 \\
\hline 0.9 & 64.37 & 88.68 & 187.53 \\
\hline \multicolumn{3}{|c|}{$\gamma_{0}=0.3$} \\
\hline$\tau$ & $n_{0}=5$ \\
\hline 0.5 & 2.14 & 2.60 & 52.15 \\
\hline
\end{tabular}

\begin{tabular}{|c|c|c|c|}
\hline 0.6 & 4.18 & 5.59 & 87.72 \\
\hline 0.7 & 10.29 & 15.68 & 135.30 \\
\hline 0.8 & 28.47 & 81.93 & 198.12 \\
\hline 0.9 & 108.36 & 204.42 & 277.14 \\
\hline & \multicolumn{3}{|c|}{$n_{0}=10$} \\
\hline 0.5 & 1.14 & 1.19 & 5.88 \\
\hline 0.6 & 1.43 & 1.62 & 15.94 \\
\hline 0.7 & 2.57 & 3.18 & 39.28 \\
\hline 0.8 & 8.97 & 17.28 & 86.08 \\
\hline 0.9 & 66.80 & 90.43 & 190.22 \\
\hline \multicolumn{3}{|c|}{$\gamma_{0}=0.5$} \\
\hline$\tau$ & $n_{0}=5$ \\
\hline 0.5 & 2.23 & 2.84 & 55.84 \\
\hline 0.6 & 4.55 & 6.70 & 93.57 \\
\hline 0.7 & 13.62 & 19.89 & 142.81 \\
\hline 0.8 & 10.88 & 98.25 & 204.75 \\
\hline 0.9 & 115.21 & 214.12 & 282.74 \\
\hline \multicolumn{3}{|c|}{$n_{0}=10$} \\
\hline 0.5 & 1.16 & 1.22 & 7.08 \\
\hline 0.6 & 1.52 & 1.71 & 18.55 \\
\hline 0.7 & 2.71 & 3.78 & 44.45 \\
\hline 0.8 & 9.08 & 20.17 & 96.26 \\
\hline 0.9 & 69.43 & 98.72 & 197.42 \\
\hline
\end{tabular}

Table 2. ATS ${ }_{1}$ values of the DVP MCV, DVSSI MCV and DSH MCV charts when $p=3, n_{0} \in\{5,10\}$, $\gamma_{0} \in\{0.1,0.3,0.5\}, h_{S}=0.1$ and $\mathrm{ATS}_{0}=370$.

\begin{tabular}{|c|c|c|c|}
\hline & DVP MCV & DVSSI MCV & DSH MCV \\
\hline & \multicolumn{3}{|c|}{$\gamma_{0}=0.1$} \\
\hline$\tau$ & \multicolumn{3}{|c|}{$n_{0}=5$} \\
\hline 0.5 & 3.67 & 5.24 & 93.38 \\
\hline 0.6 & 8.12 & 12.79 & 135.17 \\
\hline 0.7 & 18.35 & 37.64 & 183.46 \\
\hline 0.8 & 42.47 & 142.73 & 237.73 \\
\hline 0.9 & 125.64 & 259.18 & 302.76 \\
\hline & \multicolumn{3}{|c|}{$n_{0}=10$} \\
\hline 0.5 & 1.17 & 1.24 & 8.22 \\
\hline 0.6 & 1.52 & 1.76 & 20.33 \\
\hline 0.7 & 2.82 & 3.78 & 44.02 \\
\hline 0.8 & 9.70 & 21.43 & 101.49 \\
\hline 0.9 & 70.58 & 102.47 & 202.16 \\
\hline & \multicolumn{3}{|c|}{$\gamma_{0}=0.3$} \\
\hline$\tau$ & \multicolumn{3}{|c|}{$n_{0}=5$} \\
\hline 0.5 & 4.53 & 5.42 & 96.66 \\
\hline 0.6 & 9.34 & 13.29 & 139.23 \\
\hline 0.7 & 19.98 & 38.57 & 186.39 \\
\hline 0.8 & 44.66 & 145.04 & 241.67 \\
\hline 0.9 & 128.21 & 260.56 & 304.71 \\
\hline & \multicolumn{3}{|c|}{$n_{0}=10$} \\
\hline 0.5 & 1.19 & 1.27 & 8.46 \\
\hline 0.6 & 1.61 & 1.93 & 21.05 \\
\hline 0.7 & 2.97 & 3.94 & 48.60 \\
\hline 0.8 & 10.26 & 23.21 & 103.33 \\
\hline 0.9 & 73.62 & 110.39 & 203.71 \\
\hline & \multicolumn{3}{|c|}{$\gamma_{0}=0.5$} \\
\hline$\tau$ & \multicolumn{3}{|c|}{$n_{0}=5$} \\
\hline 0.5 & 4.94 & 6.36 & 103.00 \\
\hline
\end{tabular}




\begin{tabular}{|c|c|c|c|}
\hline 0.6 & 10.67 & 15.97 & 146.12 \\
\hline 0.7 & 21.81 & 46.98 & 194.52 \\
\hline 0.8 & 47.18 & 164.27 & 248.88 \\
\hline 0.9 & 135.79 & 267.85 & 309.54 \\
\hline & \multicolumn{3}{|c|}{$n_{0}=10$} \\
\hline 0.5 & 1.21 & 1.30 & 9.88 \\
\hline 0.6 & 1.72 & 1.95 & 24.26 \\
\hline 0.7 & 3.13 & 4.70 & 54.47 \\
\hline 0.8 & 13.99 & 25.92 & 111.62 \\
\hline 0.9 & 77.80 & 113.17 & 210.63 \\
\hline
\end{tabular}

Table 3. ATS $\mathrm{S}_{1}$ values of the DVP MCV, DVSS MCV and DVSI MCV charts when $p=2, n_{0} \in\{5,10\}$, $\gamma_{0} \in\{0.1,0.3,0.5\}, h_{S}=0.1$ and $\mathrm{ATS}_{0}=370$.

\begin{tabular}{|c|c|c|c|}
\hline & DVP MCV & DVSS MCV & DVSI MCV \\
\hline & \multicolumn{3}{|c|}{$\gamma_{0}=0.1$} \\
\hline$\tau$ & \multicolumn{3}{|c|}{$n_{0}=5$} \\
\hline 0.5 & 2.09 & 4.69 & 6.18 \\
\hline 0.6 & 3.90 & 7.11 & 12.11 \\
\hline 0.7 & 9.41 & 17.71 & 32.55 \\
\hline 0.8 & 25.80 & 83.49 & 85.43 \\
\hline \multirow[t]{2}{*}{0.9} & 102.31 & 227.32 & 205.96 \\
\hline & \multicolumn{3}{|c|}{$n_{0}=10$} \\
\hline 0.5 & 1.13 & 2.02 & 1.46 \\
\hline 0.6 & 1.40 & 2.74 & 2.47 \\
\hline 0.7 & 2.40 & 5.86 & 5.31 \\
\hline 0.8 & 8.01 & 26.15 & 23.11 \\
\hline \multirow[t]{2}{*}{0.9} & 64.37 & 147.58 & 105.16 \\
\hline & \multicolumn{3}{|c|}{$\gamma_{0}=0.3$} \\
\hline$\tau$ & \multicolumn{3}{|c|}{$n_{0}=5$} \\
\hline 0.5 & 2.14 & 4.72 & 6.29 \\
\hline 0.6 & 4.18 & 7.98 & 12.77 \\
\hline 0.7 & 10.29 & 18.12 & 33.33 \\
\hline 0.8 & 28.47 & 87.80 & 86.90 \\
\hline \multirow[t]{2}{*}{0.9} & 108.36 & 256.49 & 208.42 \\
\hline & \multicolumn{3}{|c|}{$n_{0}=10$} \\
\hline 0.5 & 1.14 & 2.03 & 1.51 \\
\hline 0.6 & 1.43 & 2.93 & 2.52 \\
\hline 0.7 & 2.57 & 6.23 & 6.03 \\
\hline 0.8 & 8.97 & 27.34 & 24.43 \\
\hline \multirow[t]{2}{*}{0.9} & 66.80 & 154.59 & 107.41 \\
\hline & \multicolumn{3}{|c|}{$\gamma_{0}=0.5$} \\
\hline$\tau$ & \multicolumn{3}{|c|}{$n_{0}=5$} \\
\hline 0.5 & 2.23 & 5.11 & 6.86 \\
\hline 0.6 & 4.55 & 8.98 & 14.71 \\
\hline 0.7 & 13.62 & 22.74 & 38.87 \\
\hline 0.8 & 10.88 & 104.09 & 96.18 \\
\hline \multirow[t]{2}{*}{0.9} & 115.21 & 266.05 & 224.61 \\
\hline & \multicolumn{3}{|c|}{$n_{0}=10$} \\
\hline 0.5 & 1.16 & 2.13 & 1.62 \\
\hline 0.6 & 1.52 & 3.23 & 2.83 \\
\hline 0.7 & 2.71 & 7.46 & 7.39 \\
\hline 0.8 & 9.08 & 33.90 & 30.01 \\
\hline 0.9 & 69.43 & 166.49 & 118.35 \\
\hline
\end{tabular}

\section{Conclusion}

In this paper, a one-sided downward DVP MCV chart is proposed for enhancing downward multivariate process monitoring. The performances of the proposed chart are measured in terms of the ATS criterion based on the Markov-chain approach. The numerical comparisons reveal that the proposed DVP MCV chart outperforms the existing DVSSI MCV, DVSS MCV, DVSI MCV and DSH MCV charts, for detecting small or moderate downward MCV shifts. No attempt has been made to improve the efficiency 
of the downward multivariate process monitoring using the VP strategy in the existing literature. The existing VP MCV chart only considered monitoring the upward MCV shifts. In certain scenarios, the detection of downward MCV shifts is very important as it shows process improvement. Moreover, the proposed DVP MCV chart also can resolve the problem of ATS-biased performances. In future research, the proposed chart can be extended to the DVP MCV charts with the presence of measurement errors and estimated parameters for monitoring the downward process shifts.

\section{Acknowledgement}

This work was funded by the Ministry of Higher Education Malaysia, Fundamental Research Grant Scheme [Grant Number 203.PMGT.6711755], for the Project entitled "A New Hybrid Model for Monitoring the Multivariate Coefficient of Variation in Healthcare Surveillance".

\section{References}

[1] T. Whelan, P. Siqueira, Coefficient of variation for use in crop area classification across multiple climates, International Journal of Applied Earth Observation and Geoinformation, Vol. 6, pp. 114-122, 2018.

[2] E.U. Weber, On the coefficient of variation as a predictor of risk sensitivity: Behavioral and neural evidence for the relative encoding of outcome variability, Journal of Mathematical Psychology, Vol. 54, pp. 395-399, 2010.

[3] S. Chanda, Y. Kanke, M. Dalen, J. Hoy, B. Tubana, Coefficient of variation from vegetarian index for sugarcane population and stalk evaluation, Agrosystems, Geosciences \& Environment, Vol. 1, pp. 1-9, 2018.

[4] R. Calif, T. Soubdhan, On the use of the coefficient of variation to measure spatial and temporal correlation of global solar radiation, Renewable Energy, Vol. 88, pp. 192-199, 2016.

[5] T. Mahmood, S.A. Abbasi, Efficient monitoring of coefficient of variation with an application to chemical reactor process, Quality and Reliability Engineering International, Vol. 37, pp. 1135-1149, 2021.

[6] C.W. Kang, M.S. Lee, Y.J. Seong, D.M. Hawkins, A control chart for the coefficient of variation, Journal of Quality Technology, Vol. 39, pp. 151-158, 2007.

[7] K.W. Khaw, X.Y. Chew, A re-evaluation of the run rules control chart for monitoring the coefficient of variation, Statistics, Optimization
\& Information Computing, Vol. 7, pp. 716-730, 2019.

[8] S.L. Lim, W.C. Yeong, M.B.C. Khoo, Z.L. Chong, K.W. Khaw, An alternative design for the variable sample size coefficient of variation chart based on the median run length and expected median run length, International Journal of Industrial Engineering: Theory, Applications and Practice, Vol. 26, pp. 199-220, 2019.

[9] R. Chen, L. Jin, Z. Li, J. Zhang. A progressive approach for the detection of the coefficient of variation, Quality and Reliability Engineering International, 2021. DOI: https://doi.org/10.1002/qre.2877

[10] R. Chen, Z. Li, J. Zhang, A generally weighted moving average control chart for monitoring the coefficient of variation, Applied Mathematical Modelling, Vol. 70, pp. 190-205, 2019.

[11] W.C. Yeong, M.B.C. Khoo, W.L. Teoh, P. Castagliola, A control chart for the multivariate coefficient of variation, Quality and Reliability Engineering International, Vol. 32, pp. 12131225, 2016.

[12] K.W. Khaw, M.B.C. Khoo, P. Castagliola, M.A. Rahim, New adaptive control charts for monitoring the multivariate coefficient of variation, Computers \& Industrial Engineering, Vol. 126, pp. 595-610, 2018.

[13] K.W. Khaw, X.Y. Chew, W.C. Yeong, S.L. Lim, Optimal design of the synthetic control chart for monitoring the multivariate coefficient of variation, Chemometrics and Intelligent Laboratory Systems, Vol. 186, pp. 33-40, 2019

[14] Q.T. Nguyen, K.P. Tran, H.L. Heuchenne, T.H. Nguyen, H.D. Nguyen, Variable sampling interval Shewhart control chart for monitoring the multivariate coefficient of variation, Applied Stochastic Models in Business and Industry, Vol. 35, pp. 1253$1268,2019$.

[15] X.Y. Chew, K.W. Khaw, One-sided downward control chart for monitoring the multivariate coefficient of variation with VSSI strategy, Journal of Mathematical and Fundamental Sciences, Vol. 52, pp. 112-130, 2020.

[16] X.Y. Chew, M.B.C. Khoo, K.W. Khaw, W.C. Yeong, Z.L. Chong, A proposed variable parameter control chart for monitoring the multivariate coefficient of variation, Quality and Reliability Engineering International, Vol. 35, pp. 2442-2461, 2019.

[17] V. Giner-Bosch, K.P. Tran, P. Castagliola, M.B.C. Khoo, An EWMA control chart for the multivariate coefficient of variation, Quality 
and Reliability Engineering International, Vol. 35, pp. 1515-1541, 2019.

[18] H.N. Ayyoub, M.B.C. Khoo, S. Saha, M.H. Lee. Variable sampling interval EWMA chart for multivariate coefficient of variation, Communications in Statistics - Theory and Methods, 2021. DOI: https://doi.org/10.1080/03610926.2020.181810 0

[19] W.C. Yeong, S.L. Lim, M.B.C. Khoo, P. Castagliola, Monitoring the coefficient of variation using a variable parameter chart, Quality Engineering, Vol. 30, pp. 212-235, 2018.

[20] Y. Lin, The variable parameter $\bar{X}$ control charts for monitoring autocorrelated processes, Communications in Statistics - Simulation and Computation, Vol. 38, pp. 729-749, 2009.

[21] Y. Lin, C. Chou, Non-normality and the variable parameters $\bar{X}$ control charts, European Journal of Operational Research, Vol. 176, pp. 361-373, 2007.

[22] Z. Guo, L. Cheng, Z. Lu, Economic design of the variable parameters $\bar{X}$ control chart with a correlated A\&L switching rule. Quality and Reliability Engineering International, Vol. 30, pp. 235-246, 2014.

[23] R. Wang, X. Fu, J. Yuan, Z. Dong, Economic design of variable parameter $\bar{X}$ Shewhart control chart used to monitor continuous production. Quality Technology \& Quantitative Management, Vol. 15, pp. 106-124, 2018.

[24] M.H. Lee, M.B.C. Khoo, X.y. Chew, Economic -statistical designof variable parameter $S$ chart, Quality Technology \& Quantitative Management, Vol. 17, pp. 580-591 2020.

[25] N.L. Chong, M.B.C. Khoo, P. Castagliola, S. Saha, F.N. Min, A variable parameters auxiliary information based quality control chart with application in a spring manufacturing process: The Markov chain approach, Quality Engineering, 2021. DOI: https://doi.org/10.1080/08982112.2020.183041 7

[26] H. Sabahno, P. Castagliola, A. Amiri, A variable parameters multivariate control chart of the process mean and variability with measurement errors, Quality and Reliability Engineering International, Vol. 36, pp. 1161-1196, 2020.

[27] V.G. Voinov, M.S. Nikulin, Unbiased estimator and their applications, multivariate case (2nd edn), Kluwer, Dordrecht, 1996.

[28] A.N.B. Muhammad, W.C. Yeong, Z.L. Chong, S.L. Lim, M.B.C. Khoo, Monitoring the coefficient of variation chart using a variable sample size EWMA chart, Computers \& Industrial Engineering, Vol. 126, pp. 378-398, 2018.

\section{Creative Commons Attribution License 4.0 (Attribution 4.0 International, CC BY 4.0)}

This article is published under the terms of the Creative Commons Attribution License 4.0 https://creativecommons.org/licenses/by/4.0/deed.en US 